\title{
A Generalized Lorenz System
}

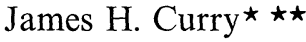

Advanced Study Program, National Center for Atmospheric Research ${ }^{\star \star \star}$, Boulder, Colorado 80307, USA

\begin{abstract}
A 14-dimensional generalized Lorenz system of ordinary differential equations is constructed and its bifurcation sequence is then studied numerically. Several fundamental differences are found which serve to distinguish this model from Lorenz's original one, the most unexpected of which is a family of invariant two-tori whose ultimate bifurcation leads to a strange attractor. The strange attractor seems to have many of the gross features observed in Lorenz's model and therefore is an excellent candidate for a higher dimensional analogue.
\end{abstract}

\section{Introduction}

In the Boussinesq approximation the equations governing atmospheric convection can be written in the following dimensionless form:

$$
\begin{aligned}
& (\Delta \psi)_{t}=-\frac{\partial(\psi, \Delta \psi)}{\partial(x, z)}+\sigma \Delta^{2} \psi+\sigma \frac{\partial \theta}{\partial x} \\
& (\theta)_{t}=-\frac{\partial(\psi, \theta)}{\partial(x, z)}+R \frac{\partial \psi}{\partial x}+\Delta \theta
\end{aligned}
$$

where $\psi(x, z, t)$ and $\theta(x, z, t)$ denote the stream function and departure of temperature from a linear profile and $\sigma$ and $R$ denote the Prandtl and Rayleigh numbers respectively. For a derivation of the equations in the form (1) and a discussion of boundary conditions we refer the reader to [1].

In [7], Lorenz used the above system of equations as a starting point for his study on the predictability of certain atmospheric flows. By assuming that $\psi(x, z, t)$ and $\theta(x, z, t)$ had a particular form it was possible to pass from (1) to a system of three quadratically coupled ordinary differential equations, this system of equations has since become known as the Lorenz system. The Lorenz system was the

* On leave from Department of Mathematics, Howard University, Washington, DC, USA

$\star \star$ Current address: Department of Meterology, M.I.T., Cambridge, MA, USA

$\star \star \star$ The National Center for Atmospheric Research is sponsored by the National Science Foundation 
first example of a physically interesting system of equation which was observed to have a strange attractor.

In recent papers McLaughlin [9] and McLaughlin and Martin [10] have considered various models of the convection process. In [10] they proved that subcritical instabilities exist in the model studied by Lorenz, and in [9] McLaughlin considered the restricted three-dimensional convection problem and observed a sequence of bifurcations leading to stochastic behavior; preceding stochastic behavior he found periodic states and phase locking, provided that one component was sufficiently energetic.

Studies of the Lorenz system which concentrate more on its mathematical structure can be found in [2-5], [13], and [14]. The work in these articles is based, at least in part, on the results of extensive numerical experiments, and even though there is little doubt that what one observes in the computer is in qualitative agreement with the actual behavior of solutions to Lorenz's equations, proofs are still lacking.

This study is also motivated by the equations of Lorenz as were the works cited above; however, we are more concerned with the question of the extent to which the qualitative properties discovered by Lorenz in his three-component system are reproducible in a larger system. Further, if higher dimensional analogues exist then there is no a priori reason to believe that the bifurcation sequence should parallel what is observed in the smaller system.

Unfortunately, because there are no proofs available one must resort to numerical experiments to provide partial answers to questions like those above. Moreover, once a model has been chosen, many of the comments made above will be equally applicable to the new truncation. The way out of this dilemma is clear but not yet realized.

\section{Generalized Lorenz Systems}

In what follows we shall let $\Lambda$ denote a finite non-empty subset of the lattice of non-negative integers. The truncation associated with $\Lambda$ is the system of ordinary differential equations for the time-dependent terms $\psi_{m n}(t), \theta_{m n}(t)$ obtained by the substitution of $\psi(x, z, t)$ and $\theta(x, z, t)$ of the following form into Equation (1)

$$
\begin{aligned}
& \Psi(x, z, t)=\sum_{\substack{(m, n) \in \Lambda \\
m \neq 0}} \psi_{m n}(t) \sin (\operatorname{am} x) \sin (n z) \\
& \theta(x, z, t)=\sum_{(m, n) \in \Lambda} \theta_{m n}(t) \cos (a m x) \sin (n z) .
\end{aligned}
$$

It is easily checked that by assuming that $\psi$ and $\theta$ are as defined above, the truncation associated with $\Lambda$ will be invariant under the symmetry which carries the components $\left(\psi_{m n}(t), \theta_{m n}(t)\right)$ into $\left(-\psi_{m n}(t),-\theta_{m n}(t)\right)$ whenever $m$ and $n$ are both odd and leaves them fixed when $m$ and $n$ are otherwise.

We may write the truncation associated with $\Lambda$ symbolically as

$$
\frac{d x}{d t}=X_{\Lambda}(x, R, \sigma) \text {, }
$$


where $x$ is a vector composed of the time-dependent components from (2) and $R$ and $\sigma$ are as previously defined. If we regard the ensemble of all solutions to (3) as a "phase fluid", then the fluid velocity at any point is just $X_{\Lambda}(x, R, \sigma)$. A straightforward computation yields that the divergence of the right-hand side of (3) is given by

$$
\operatorname{div} X_{\Lambda}(x, R, \sigma)=-(\sigma+1) \sum_{(m, n) \in \Lambda} \varrho_{m n} .
$$

$\varrho_{m n}=m^{2} a^{2}+n^{2}$ and $a=1 / \sqrt{2}$. Since the divergence of the vector field is always negative, it follows that any finite cell of phase fluid must tend to zero volume exponentially fast, with exponent the right-hand side of (4), as $t \rightarrow \infty$.

Another interesting fact which has been established in [3] is that all solution curves to (3) associated with any $\Lambda$ asymptotically approach zero for Rayleigh numbers less than the critical Rayleigh number. Where the critical Rayleigh number is given by

$$
R_{c}=\inf _{\substack{(m, n) \in \Lambda \\ m \neq 0}} \frac{\varrho_{m n}^{3}}{(m a)^{2}} .
$$

A somewhat less general fact has been established in [1]. There sufficient conditions have been given for a large class of truncations to have only bounded solutions.

The above comments are valid for a large class of $\Lambda$. We are interested in a subcollection of this class. Specifically, it can be seen from Section 5 of [7] that the Lorenz system, when suitably scaled, is the truncation associated with the set $\{(0,2),(1,1)\}$, hence by a generalized Lorenz system we shall mean the truncation associated with any $\Lambda$ which contains the set $\{(0,2),(1,1)\}$ as a proper subset.

\section{The Model}

In the remainder of this article we shall restrict the discussion to a particular 14component system. Specifically, we study the truncation associated with the following set:

$$
\Lambda=\{(0,2),(1,1),(2,2),(1,3),(3,1),(3,3),(2,4),(0,4)\} .
$$

In Appendix A we provide a listing of this truncation. The motivation for the above choice of $\Lambda$ is twofold:

a) It is clearly an example of a generalized Lorenz system as defined in the previous section, and

b) it was also the largest such truncation which could be conveniently handled in the machine on which many preliminary experiments were done.

Two bits of information are now readily apparent once we have fixed $\Lambda$. Namely,

$$
\operatorname{div} X_{\Lambda}=-74(\sigma+1)
$$

and the critical Rayleigh number is 6.75 . If we now choose $\sigma=10$, basing our choice solely on [7], then if $V_{0}$ is some initial volume of phase fluid, after $t$ units of time the volume will be smaller by a factor of $\left(1.27 \times 10^{-503 t}\right)$. 
In an effort to bring our notation into agreement with [7] we redefine our bifurcation parameter, $R$, to be

$$
r=\frac{R}{6.75}
$$

Though the results of [1] do not immediately extend to the present model, all of our numerical experiments indicate that the solution curve remains bounded for all times. Finally, we shall concentrate on the behavior of the Lorenz components, i.e., the triple $\psi_{11}(t), \theta_{11}(t)$, and $\theta_{02}(t)$ when describing our numerical results.

\section{Numerical Results}

In reporting the results of our numerical experiments we find it natural to divide the parameter range into three intervals. The motivation for the division is that in each interval the behavior is more complex than in the preceding one. In defining the various intervals we are not asserting that the boundaries are exact, but simply provide adequate points of separation between areas of different qualitative behavior.

The intervals are:

$$
\begin{array}{ll}
\text { Interval I } & 1 \leqq r \leqq 44.60, \\
\text { Interval II } & 44.60<r<45.10, \\
\text { Interval III } & r>45.10
\end{array}
$$

\section{a) Interval I}

As $r$ exceeds one the origin becomes unstable and a symmetric pair of stable fixed points bifurcate off; we label them $C_{1}$ and $C_{2}$. Further, as $r$ crosses the sequence of values defined by

$$
\frac{\varrho_{m n}^{3}}{(6.75)(m a)^{2}}
$$

additional symmetric pairs of stationary solutions leave the origin. However, these new fixed points all have one-dimensional unstable manifolds.

For $r \in(1,43.48)$ the model exhibits very simple dynamical behavior. There are at most seven fixed points, only two of which are stable, $C_{1}$ and $C_{2}$; further given any initial conditions in which the Lorenz components are nonzero, we find that the solution curve converges to either $C_{1}$ or $C_{2}$. For this range of $r$, the remaining critical points seem to play no significant role in the dynamics.

When $r$ passes 43.48 the $C_{i}$ become unstable by having a pair of complex conjugate eigenvalues cross into the open right half-plane, and the stability of each stationary solution is transferred to an attracting closed orbit. For $r=43.70$ the only complex eigenvalues of $C_{i}$ having positive real part are $\lambda=0.042 \pm 35.672 i$. It follows from the Hopf Bifurcation Theorem [8] that we should expect the period of our closed orbits to be close to $\frac{2 \pi}{|\lambda|} \approx 0.176$. 


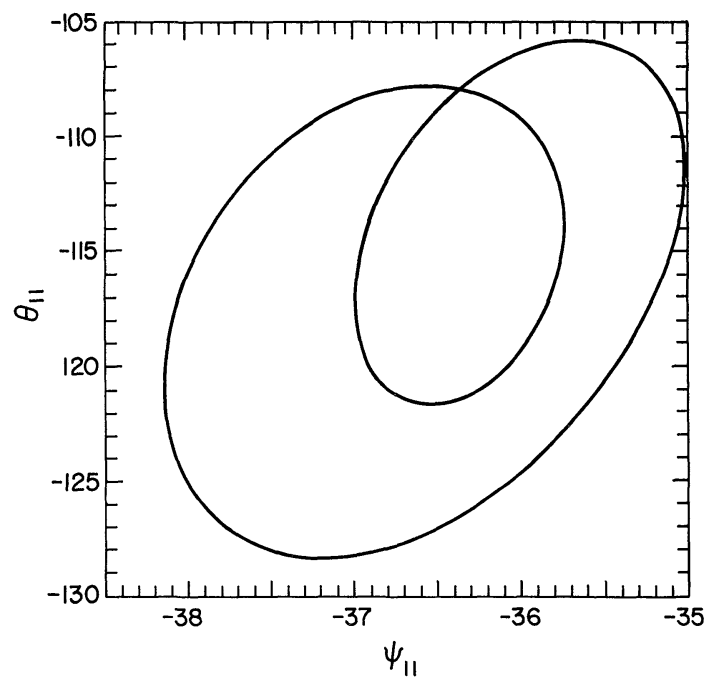

Fig. 1. $\left(\psi_{11}, \theta_{11}\right)$ phase projections for $r=44.45$

We find that the computed period is 0.155 or approximately a $12 \%$ departure from its theoretical value. The periodic orbits must be attracting since they were found by only numerically solving the equations of motion.

Another interesting value of $r$ is 44.07 . For this value the closed orbits shed by $C_{i}$ become unstable and transfer its stability to a closed orbit having twice the period.

In Figure 1 we see a projection of this new closed orbit onto the $\left(\psi_{11}, \theta_{11}\right)$ plane, its period is 0.3067 and $r=44.45$. For this value of $r$ it is quite clear that the closed orbit coming from the normal Hopf bifurcation when $r=43.48$ has indeed undergone a further bifurcation.

\section{b) Intervall II}

We denote by $\gamma$ a closed orbit, $\Sigma$ a 13-dimensional local section transverse to $\gamma$, $x \in \gamma$, and $P_{r}(x)$ the associated Poincaré map [6], [8].

In Section $3 b$ of their fundamental paper [11], Ruelle and Takens describe what one should generically expect if the spectrum of the Poincaré map consists of only a finite number of isolated eigenvalues having modulus one and a part which is contained in the open unit disc. We are interested in their Case 3. There the set of eigenvalues having modulus one consists of $\alpha$ and its conjugate $\bar{\alpha}$, which are assumed to be distinct. As $r$ exceeds some $r^{\prime}$ the eigenvalues $\alpha$ and $\bar{\alpha}$ cross the unit circle and one expects that there is an attracting invariant circle for the Poincare map, $P_{r}$, and hence an attracting torus for the flow in a neighborhood of $\gamma$.

It is not difficult to define a Poincaré map using the computer. $\Sigma$ was chosen to be the section defined by $\theta_{02}=-295$, and the solution curve was required to intersect $\Sigma$ to an accuracy of $10^{-10}$. We initially concentrate on the behavior of the flow in a neighborhood of $C_{1}$. 


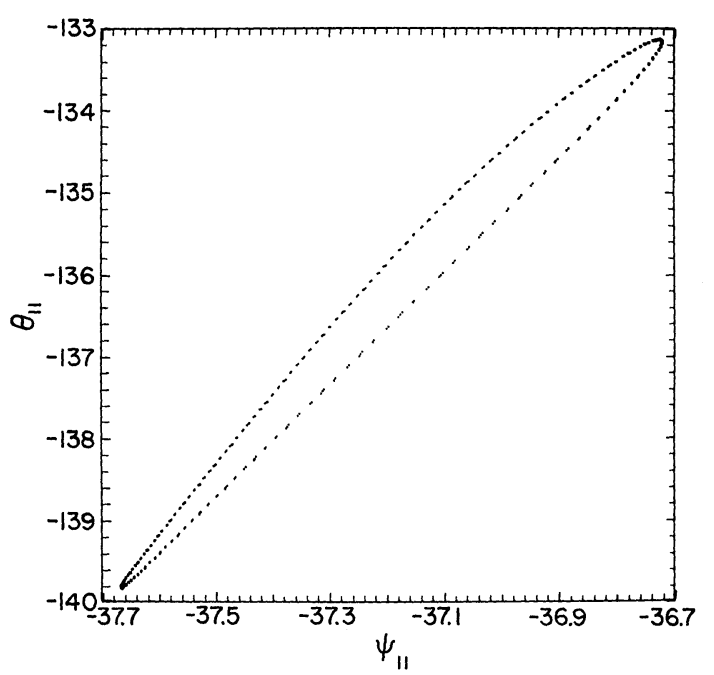

Fig. 2. $\Sigma$ projected onto the $\left(\psi_{11}, \theta_{11}\right)$ plane $r=44.88$

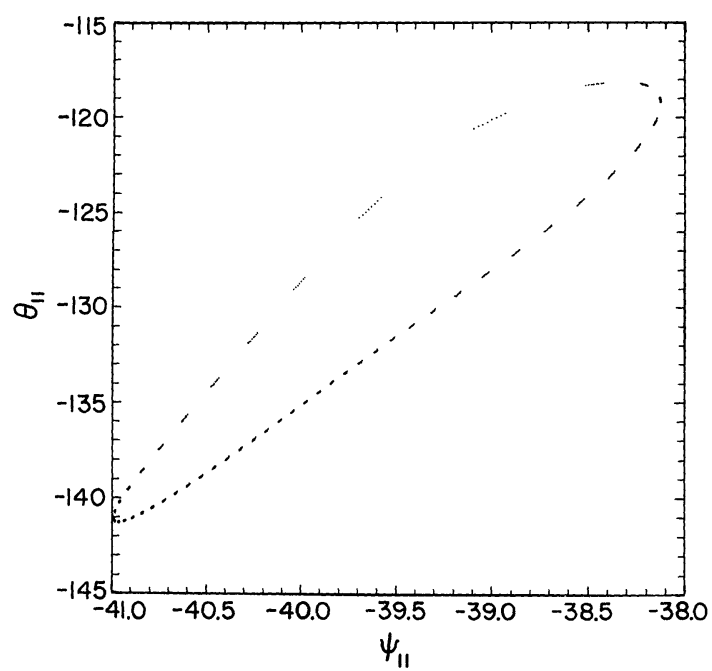

Fig. 3. $\Sigma$ projected onto the $\left(\psi_{11}, \theta_{11}\right)$ plane $r=45.06$

In Figures 2 and 3 we have the projection of $\Sigma$ onto the $\left(\psi_{11}, \theta_{11}\right)$ plane for $r$ $=44.88$ and 45.06 , respectively. These projections provide very strong evidence that the closed orbit found in interval I when $r=44.45$ has undergone a bifurcation leading to an invariant two-torus. In both figures we are viewing the results of 400 intersections of the solution curve with our codimension-one section. We remark that the 77 other projections of $\Sigma$ onto the two-dimensional planes defined by the pairs of components of our equations give qualitatively similar results. We shall denote the family of tori in a neighborhood of $C_{1}$ by $T_{1}$. 
The behavior in this parameter range is further complicated by the existence of an attracting closed orbit in the neighborhood of $C_{1}\left(C_{2}\right)$. Specifically, in the observations made above we have chosen initial conditions for the Lorenz components in a neighborhood of $C_{1}$ and for such initial conditions the solution curve converges to an attracting two-torus. If, however, we choose as initial conditions points close to the unstable manifold of the origin, then the solution curves converge to an attracting closed orbit. The existence of several attracting sets is known as hysteresis and is associated with sensitive dependence on initial conditions.

As we observed before, the 14-component model which we are studying has a normal Hopf bifurcation. The closed orbit which is shed quickly undergoes a further bifurcation which has it doubling its period. By considering the behavior of the flow only in the region surrounding $C_{1}$ we lost track of the original closed orbit.

What has happened is now apparent. The original closed orbit has continued to exist, grown in size (period $=0.3520$ ), and become stable.

\section{c) Interval III}

In Figure 4 a we see the $\Sigma$ defined in Interval II projected onto the $\left(\psi_{11}, \theta_{11}\right)$ plane when $r=45.19$. We can see the torus $T_{1}$ near the top of the figure and a sequence of points apparently moving away from it.

In order to interpret $4 \mathrm{a}$ we refer the reader to Figure $4 \mathrm{~b}$ which is the graph of $\theta_{11}$ restricted to $\Sigma, \tau$ denotes iteration of the Poincare map when starting from a point in the section. For the first 150 iterations of $P_{r}^{\tau}$ the flow remains in a neighborhood close to $T_{1}$. During the next 100 intersections of $\Sigma$ the orbit moves away from $T_{1}$ and approaches the hyperbolic closed orbit which was found to be stable for $R$ in Interval II. The solution curve then approaches $T_{1}$ again but does not appear to fall on its stable manifold. Then at a $\tau$ of 350 the flow leaves the neighborhood of $T_{1}$ only to return immediately, and once again approaches $T_{1}$. At time 480 the solution curve leaves a neighborhood of $T_{1}$, however, this excursion does not bring an immediate return to a neighborhood of its origin.

For this value of $r$ we offer the following description of the qualitative behavior of the flow. The tori $T_{1}$ and its symmetric image $T_{2}$ are marginally unstable, as are the hyperbolic closed orbits found in Interval II. Since $T_{1}$ and $T_{2}$ are marginally unstable, points which are initially sufficiently close to them will remain in that neighborhood for a significant length of time. The orbit eventually leaves the basin of attraction for $T_{1}$ along its unstable manifold which apparently lies near the stable manifold of the closed orbits mentioned above. Since the closed orbit is unstable the flow subsequently leaves along its unstable manifold which ends in a neighborhood of the stable manifold of its symmetric image. Then depending on whether or not the flow approaches sufficiently close to $T_{2}$, we see either the type of behavior described above for $T_{1}$ in Figure $4 \mathrm{a}$ or we see the unstable closed orbit push the flow back to a neighborhood of its symmetric image which lies near $T_{2}$. The entire process then repeats itself.

Finally, to complete the present section we provide Figure $5 \mathrm{a}$ and $\mathrm{b}$ which are projections of our full 14-dimensional phase space onto the $\left(\psi_{11}, \theta_{11}\right)$ and $\left(\theta_{11}, \theta_{02}\right)$ 

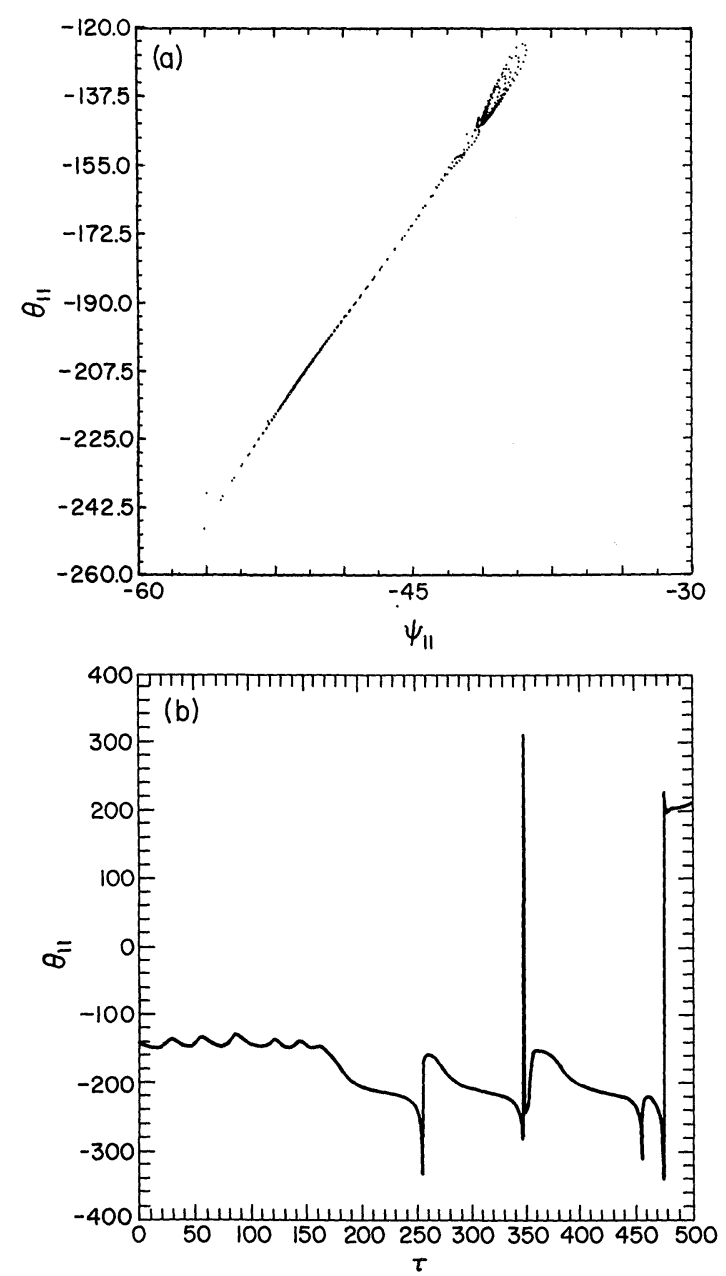

Fig. 4. a A neighborhood of the unstable torus restricted to $\Sigma$ for $r=45.19$. The torus is unstable and the flow moves away on its unstable manifold. b Time series for $\theta_{11}$ restricted to $\Sigma$ for $r=45.19$

planes, respectively, $r=45.92$. The discussion of the previous paragraph applies to these figures.

These projections provide evidence for the existence of a Lorenz-type attractor for this interval of $r$ values. Further for $r$ as great as 100 the qualitative behavior of the flow is similar to that pictured in Figure 5a and $b$.

\section{Discussion}

In this paper we have presented the results of our numerical experiments on a 14component dynamical system coming from the equations of atmospheric convection. The purpose of this study was to investigate the bifurcation sequence of a 

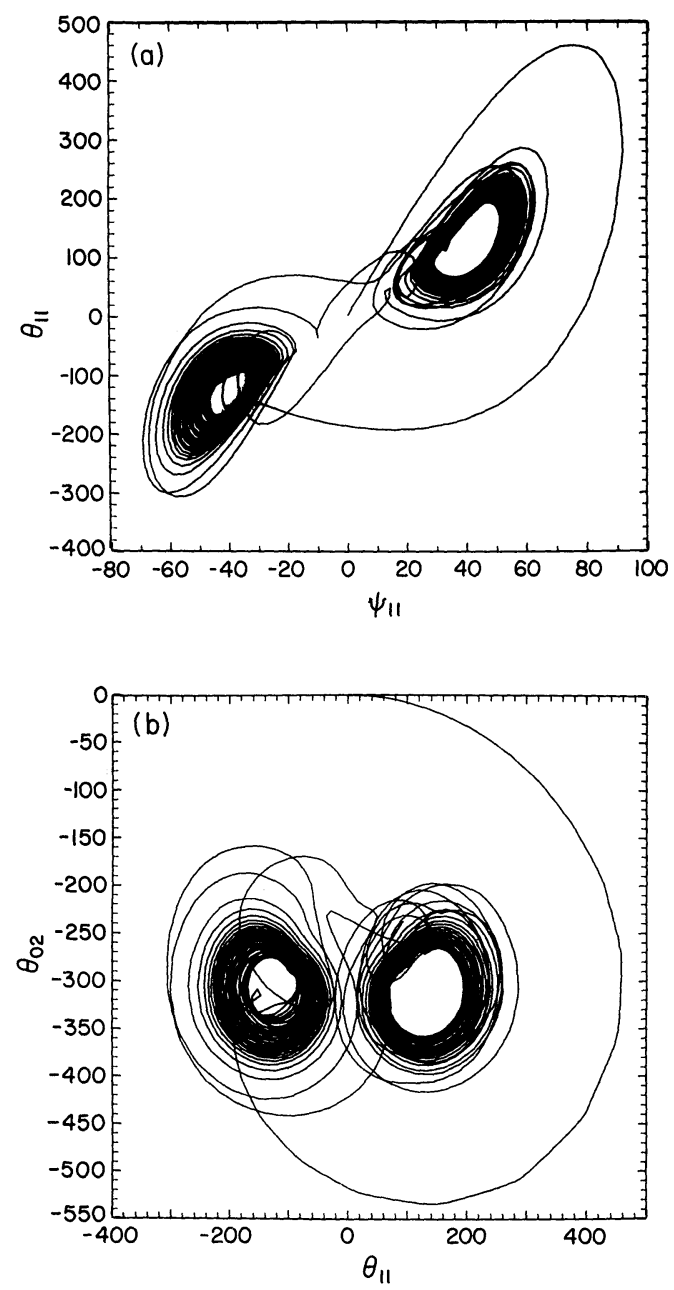

Fig. 5. a $\left(\psi_{11}, \theta_{11}\right)$ phase plot starting from a neighborhood of 0 for $r=45.92$. b $\left(\theta_{11}, \theta_{02}\right)$ phase plot starting from a neighborhood of 0 for $r=45.92$

generalized Lorenz system and to ascertain to what extent the behavior observed in the Lorenz system of three ordinary differential equations is reproducable by a larger truncation. We have found that unlike the Lorenz system, the model we considered exhibits a normal Hopf bifurcation. The closed orbit which is shed undergoes another bifurcation which has it transferring stability to a periodic orbit having approximately twice the period. This periodic orbit subsequently bifurcates and is replaced by an attracting torus. The torus eventually, as $r$ is increased, loses its stability. However, prior to becoming unstable we have evidence that, like the Lorenz system, our system also exhibits hysteresis, and hence for some parameter values there are several attractors present. When the torus becomes unstable it pushes the flow out of its basin of attraction and into a neighborhood of its 
symmetric image, which is also unstable (Fig. 5a and b). Hence the process must repeat itself. These apparently arbitrary transitions between neighborhoods of $T_{1}$ and $T_{2}$ are similar to the behavior observed in the Lorenz system when $r$ exceeds 24.75. Apparently this type of behavior has been seen prior to $r=24.75$ in the Lorenz system [4]. We remark, however, that we did not observe any region of preturbulence in our model prior to the loss of stability of the stationary solutions $C_{i}$. Further, the fact that we have a normal Hopf bifurcation indicates that such a subcritical instability does not happen [8].

A fundamental difference between the Lorenz system and our 14-dimensional generalization is that the unstable stationary solutions in the smaller system have been replaced by tori in our larger system as the mechanism which drives the flow in the turbulent parameter range (see [5]).

This paper leaves several questions unanswered, fundamental among them are whether it is possible to reduce the model considered here to the study of mappings of the interval or square into itself; this model provides an example of a two-torus whose bifurcation leads to a strange attractor: are we observing motion on a three-torus; and, in general, how much of the analysis done by Guckenheimer, Lanford, Williams and Yorke can be extended to this model?

Acknowledgments. We would like to thank Professor E. N. Lorenz for several helpful discussions, and Professor O. E. Lanford III and J. A. Yorke for helpful discussions and providing preprints of their recent work.

\section{Appendix A}

In this appendix we list the fourteen component ordinary differential equations which we have studied in the main body of this paper. In what follows the dot above the variable indicates differentiation with respect to time and to allow for more compact notation we suppress the explicit dependence of the components on time.

$$
\begin{aligned}
\dot{\psi}_{11}= & -a\left\{\frac{7}{3} \psi_{13} \psi_{22}+\frac{17}{6} \psi_{13} \psi_{24}+\frac{1}{3} \psi_{31} \psi_{22}+\frac{9}{2} \psi_{33} \psi_{24}\right\} \\
& -\sigma \frac{3}{2} \psi_{11}+\sigma a \frac{2}{3} \theta_{11}, \\
\dot{\psi}_{13}= & a\left\{-\frac{9}{19} \psi_{11} \psi_{22}+\frac{33}{38} \psi_{11} \psi_{24}+\frac{2}{19} \psi_{31} \psi_{22}-\frac{125}{38} \psi_{31} \psi_{24}\right\} \\
& -\sigma \frac{19}{2} \psi_{13}+\sigma a \frac{2}{19} \theta_{13}, \\
\dot{\psi}_{22}= & a\left\{\frac{4}{3} \psi_{11} \psi_{13}-\frac{2}{3} \psi_{11} \psi_{31}-\frac{4}{3} \psi_{13} \psi_{31}\right\}-\sigma 6 \psi_{22}+\sigma a \frac{1}{3} \theta_{22}, \\
\dot{\psi}_{31}=a\left\{\frac{9}{11} \psi_{11} \psi_{22}+\frac{14}{11} \psi_{13} \psi_{22}+\frac{85}{22} \psi_{13} \psi_{24}\right\} & \\
& -\sigma \frac{11}{2} \psi_{31}+\sigma a \frac{6}{11} \theta_{31}, \\
\dot{\psi}_{33}= & a\left\{\frac{11}{6} \psi_{11} \psi_{24}\right\}-\sigma \frac{27}{2} \psi_{33}+\sigma a \frac{2}{9} \theta_{33}, \\
\dot{\psi}_{24}= & a\left\{-\frac{2}{9} \psi_{11} \psi_{13}-\psi_{11} \psi_{33}+\frac{5}{9} \psi_{13} \psi_{31}\right\} \\
& -\sigma 18 \psi_{24}+\sigma a \frac{1}{9} \theta_{24},
\end{aligned}
$$




$$
\begin{aligned}
& \dot{\theta}_{11}=a\left\{\psi_{11} \theta_{02}+\psi_{13} \theta_{22}-\frac{1}{2} \psi_{13} \theta_{24}-\psi_{13} \theta_{02}+2 \psi_{13} \theta_{04}\right. \\
& +\psi_{22} \theta_{13}+\psi_{22} \theta_{31}+\psi_{31} \theta_{22}+\frac{3}{2} \psi_{33} \theta_{24}-\frac{1}{2} \psi_{24} \theta_{13} \\
& \left.+\frac{3}{2} \psi_{24} \theta_{33}\right\}+R a \psi_{11}-\frac{3}{2} \theta_{11} \text {, } \\
& \dot{\theta}_{13}=a\left\{-\psi_{11} \theta_{22}+\frac{1}{2} \psi_{11} \theta_{24}-\psi_{11} \theta_{02}+2 \psi_{11} \theta_{04}-\psi_{22} \theta_{11}\right. \\
& \left.-2 \psi_{31} \theta_{22}+\frac{5}{2} \psi_{31} \theta_{24}+\frac{1}{2} \psi_{24} \theta_{11}+\frac{5}{2} \psi_{24} \theta_{31}\right\} \\
& +\operatorname{Raw}_{13}-\frac{19}{2} \theta_{13} \text {, } \\
& \dot{\theta}_{22}=a\left\{\psi_{11} \theta_{13}-\psi_{11} \theta_{31}-\psi_{13} \theta_{11}+2 \psi_{13} \theta_{31}+4 \psi_{22} \theta_{04}\right. \\
& \left.-\psi_{31} \theta_{11}+2 \psi_{31} \theta_{13}-2 \psi_{24} \theta_{02}\right\}+R a 2 \psi_{22}-6 \theta_{22} \text {, } \\
& \dot{\theta}_{31}=a\left\{\psi_{11} \theta_{22}-2 \psi_{13} \theta_{22}+\frac{5}{2} \psi_{13} \theta_{24}-\psi_{22} \theta_{11}+2 \psi_{22} \theta_{13}\right. \\
& \left.+4 \psi_{31} \theta_{02}-4 \psi_{33} \theta_{02}+8 \psi_{33} \theta_{04}-\frac{5}{2} \psi_{24} \theta_{13}\right\} \\
& +R a 3 \psi_{31}-\frac{11}{2} \theta_{31} \text {, } \\
& \dot{\theta}_{33}=a\left\{\frac{3}{2} \psi_{11} \theta_{24}-4 \psi_{31} \theta_{02}+8 \psi_{31} \theta_{04}-\frac{3}{2} \psi_{24} \psi_{11}\right\} \\
& +R a 3 \psi_{33}-\frac{27}{2} \theta_{33} \text {, } \\
& \dot{\theta}_{24}=a\left\{-\frac{1}{2} \psi_{11} \theta_{13}-\frac{3}{2} \psi_{11} \theta_{33}+\frac{1}{2} \psi_{13} \theta_{11}-\frac{5}{2} \psi_{13} \theta_{31}\right. \\
& \left.-2 \psi_{22} \theta_{02}-\frac{5}{2} \psi_{31} \theta_{13}-\frac{3}{2} \psi_{33} \theta_{11}\right\}+R a 2 \psi_{24}-18 \theta_{24} \text {, } \\
& \dot{\theta}_{02}=a\left\{-\frac{1}{2} \psi_{11} \theta_{11}+\frac{1}{2} \psi_{11} \theta_{13}+\frac{1}{2} \psi_{13} \theta_{11}+\psi_{22} \theta_{24}-\frac{3}{2} \psi_{31} \theta_{31}\right. \\
& \left.+\frac{3}{2} \psi_{31} \theta_{33}+\frac{3}{2} \psi_{33} \theta_{31}+\psi_{24} \theta_{24}\right\}-4 \theta_{02} \text {, } \\
& \dot{\theta}_{04}=-a\left\{\psi_{11} \theta_{13}+\psi_{13} \theta_{11}+2 \psi_{22} \theta_{22}+4 \psi_{31} \theta_{33}+4 \psi_{33} \theta_{31}\right\} \\
& -16 \theta_{04} \text {. }
\end{aligned}
$$

Finally in all computations reported here $a=1 / \sqrt{2}$.

\section{References}

1. Curry,J.H.: Bounded solutions of finite dimensional approximations to the Boussinesq equations. SIAM J. Math. Anal. (to appear)

2. Guckenheimer,J.: A strange attractor. In : Lecture notes in applied mathematical sciences, Vol. 19, pp. 368-391. Berlin-Heidelberg-New York: Springer 1976

3. Joseph,D.D.: On the stability of the Boussinesq equations. Arch. Rat. Mech. Anal. 20, 59-71 (1965)

4. Kaplan, J., Yorke, J.A.: Preturbulence: A metastable regime in the system of Lorenz. Preprint (1976)

5. Lanford,O.E.III : Qualitative and statistical theory of dissipative systems. Preprint (1977)

6. Lanford,O.E.III : Bifurcation of periodic solutions into invariant tori; The work of Ruelle and Takens. Nonlinear problems in the physical sciences and biology. In: Lecture notes in mathematics, Vol. 322. Berlin-Heidelberg-New York: Springer 1973

7. Lorenz,E.N.: Deterministic nonperiodic flow. J. Atmos. Sci. 20, 130-141 (1963)

8. Marsden,J., McCracken, M. : The Hopf bifurcation and its application. In : Lecture notes in applied mathematical sciences, Vol. 19. Berlin-Heidelberg-New York: Springer 1976 
9. McLaughlin, J.: Successive bifurcations leading to stochastic behavior. J. Stat. Phys. 15, 307-326 (1976)

10. McLaughlin,J., Martin,P.C.: Phys. Rev. A12, 186 (1975)

11. Ruelle,D., Takens, F.: On the nature of turbulence. Commun. math. Phys. 20, 167-192 (1971)

12. Smale,S.: Differentiable dynamical systems. Bull. Am. Math. Soc. 73, 747-817 (1967)

13. Williams, R.F.: Structure of Lorenz attractors. Preprint (1976)

14. Robbin,K.A.: A new approach to subcritical instabilities and turbulent transitions in a simple dynamo. Proc. Cambridge Phil. Soc. (1977)

Communicated by J. Moser

Received February 1, 1978 